\title{
Pathfinding the Flight Advanced Stirling Convertor Design with the ASC-E3
}

The Advanced Stirling Convertor (ASC) was initially developed by Sunpower, Inc. under contract to NASA Glenn Research Center (GRC) as a technology development project. The ASC technology fulfills NASA's need for high efficiency power convertors for future Radioisotope Power Systems (RPS). Early successful technology demonstrations between 2003 to 2005 eventually led to the expansion of the project including the decision in 2006 to use the ASC technology on the Advanced Stirling Radioisotope Generator (ASRG). Sunpower has delivered 22 ASC convertors of progressively mature designs to date to GRC. Currently, Sunpower with support from GRC, Lockheed Martin Space System Company (LMSSC), and the Department of Energy (DOE) is developing the flight ASC-F in parallel with the ASC-E3 pathfinders. Sunpower will deliver four pairs of ASC-E3 convertors to GRC which will be used for extended operation reliability assessment, independent validation and verification testing, system interaction tests, and to support LMSSC controller verification. The ASC-E3 and -F convertors are being built to the same design and processing documentation and the same product specification. The initial two pairs of ASC-E3 are built before the flight units and will validate design and processing changes prior to implementation on the ASC-F flight convertors. This paper provides a summary on development of the ASC technology and the status of the ASC-E3 build and how they serve the vital pathfinder role ahead of the flight build for ASRG. The ASRG is part of two of the three candidate missions being considered for selection for the Discovery 12 mission. 


\section{Pathfinding the Flight Advanced Stirling Convertor Design with the ASC-E3}

Wayne A. Wong

NASA Glenn Research Center

Kyle Wilson, Eddie Smith, \& Josh Collins Sunpower, Inc.

International Energy Conversion Engineering

Conference, July 29 - August 1, 2012 


\section{Agenda}

- Background - ASC Technology Evolution

- ASC-E3 Objectives and Plans

- ASC-E3 and ASC-F Advancements

- ASC-E3 and ASC-F Status Highlights

- Path Forward

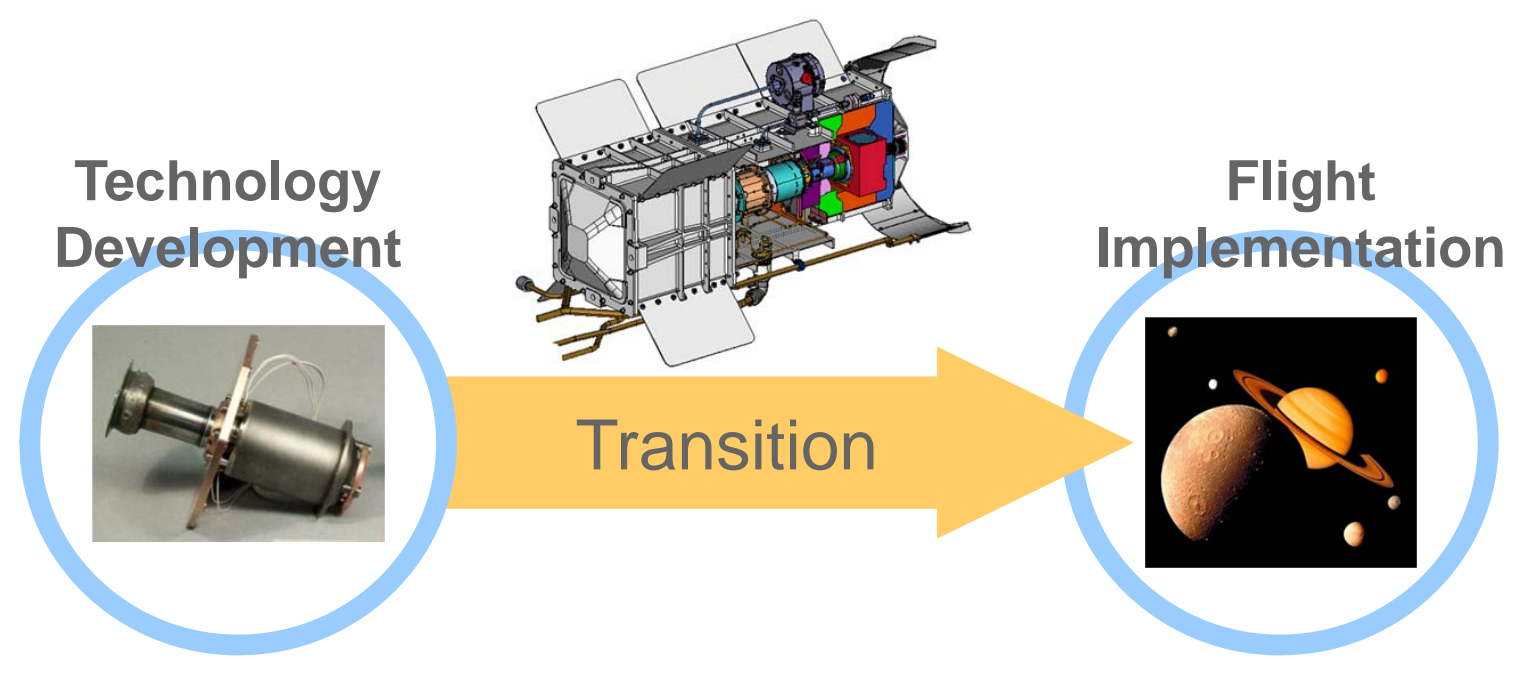




\section{ASC Development}

\section{Objectives}

- Develop efficient low mass power convertor to reduce plutonium fuel requirements for future NASA RPS

- Demonstrate ASC performance and establish long life and reliability of the design

- Prepare for flight production to support potential use of ASRG on Discovery 12 mission

\section{Approach}

- Form integrated development team: Sunpower ASC development, GRC technology development and life \& reliability assessment, Lockheed Martin (LM) flight requirements and interfaces; DOE management and overall quality assurance

- Refine the ASC through several generations of convertor builds, addressing new requirements, and implementing lessons learned

- Perform convertor-level extended operation and durability tests, and component and materials reliability assessment

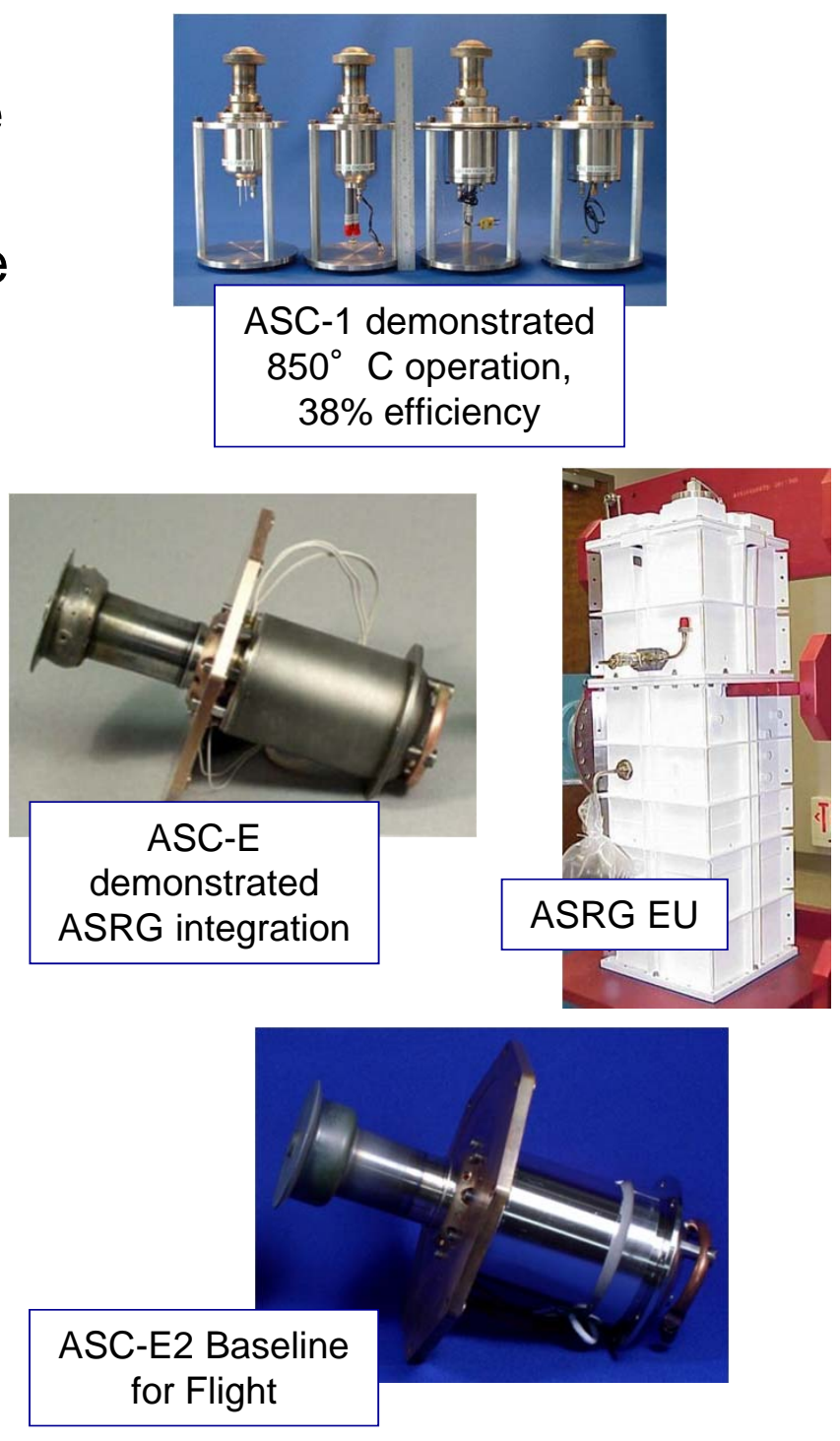




\section{Sunpower ASC Technology Evolution}

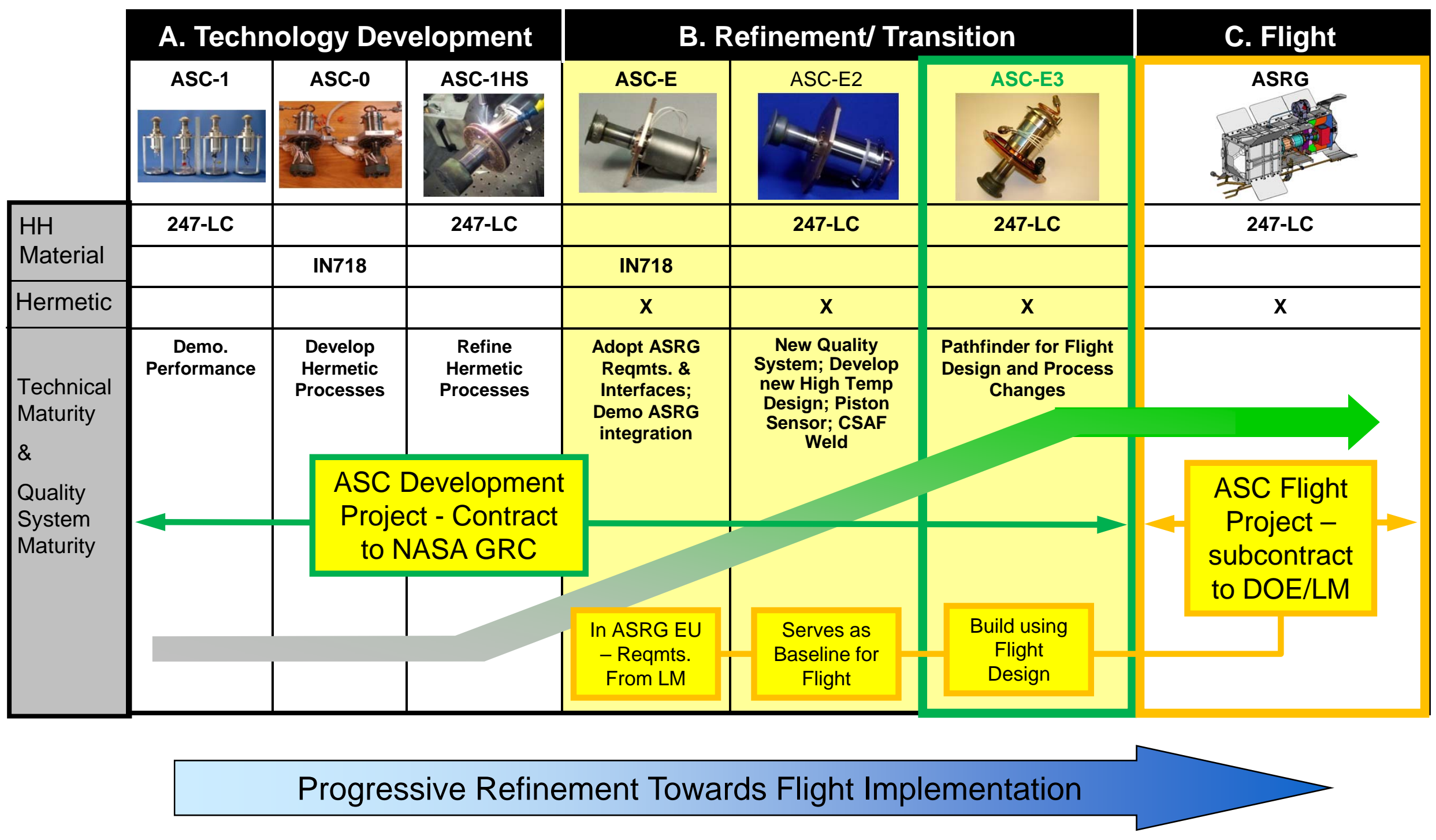




\section{ASC-E2 Baseline for Flight Design}

- Established baseline design for flight

- Eight ASC-E2 convertors delivered to GRC in 2010 for extended operation, durability testing, and for Lockheed Martin's controller development

- Significant ASC-E2 Advancements:

- New integrated 247-LC heater head design for increased creep life margin and better performance at up to $850^{\circ} \mathrm{C} \mathrm{T}$ (hot)

- E-Beam welded CSAF provides more robust metallurgical bond

- New ASC Piston Sensor (APS/ILS) eliminates two feedthroughs

- Production under new Sunpower Quality Management System

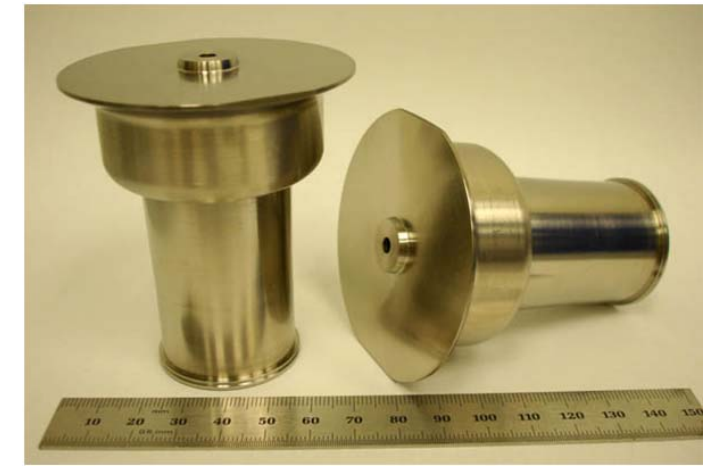

ASC-E2 Heater Head Assemblies

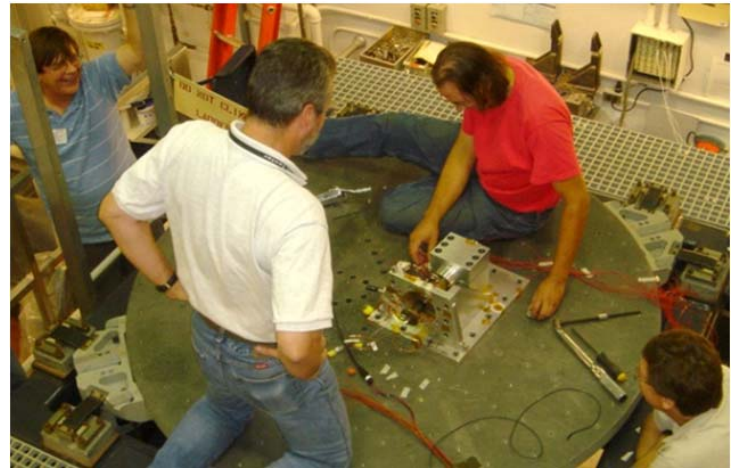

Workmanship Vibration Testing at GRC

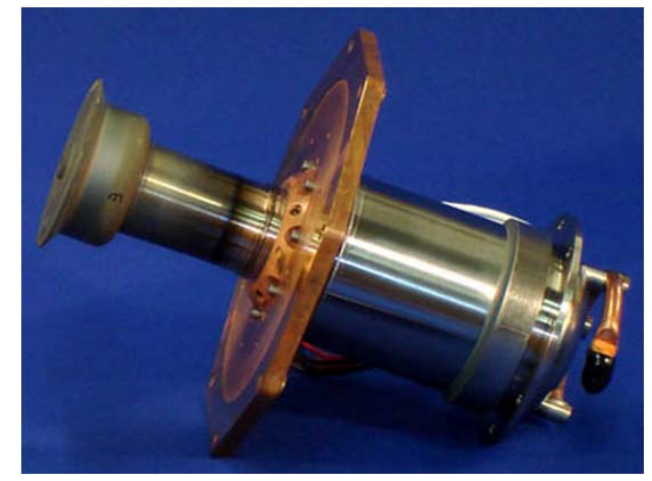

Hermetic ASC-E2 


\section{ASC-E3 Objectives}

- Demonstrate ASC-E3 production and performance - reduce risk to flight ASC-F

- ASC-E3 is pathfinders for design and process changes; Built using the ASC-F product specification and design documentation

- Joint Configuration Control Board (Sunpower, Lockheed Martin, DOE, GRC) for ASC-E3 \& Flight.

- Changes from -E2 to -E3/-F are driven by: Materials \& Processing (M\&P) flight requirements; Cleanliness and planetary protection requirements; ASRG interfaces; ASC-E2 lessons learned

- Validate Sunpower production capability in new facility including clean room, production areas, and test cells

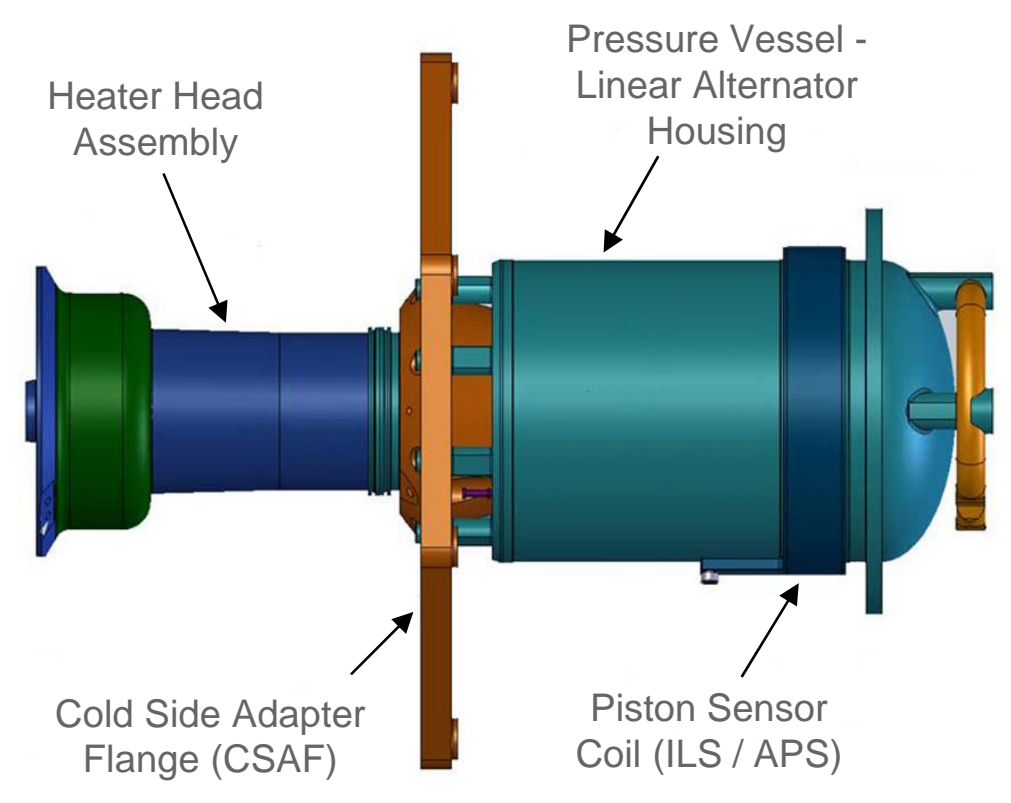

ASC - $209.5 \mathrm{~mm}$ long $\times 155.1 \mathrm{~mm}$ wide (8.25 in. $x 6.11$ in.), $2.4 \mathrm{~kg}$

The ASC-E3 build minimizes the risk to flight by serving as a pathfinder for flight design and processes 


\section{ASC-E3 Plan}

- ASC-E3 Progressive Build Plan:

- $1^{\text {st }}$ Pair - Flight design pathfinder; For Lockheed Martin controller verification

- $2^{\text {nd }}$ Pair - Clean room pathfinder; For extended operation, IV\&V, and system interaction testing

- $3^{\text {rd }}$ and $4^{\text {th }}$ Pairs - Built to approved flight documentation; For extended operation, IV\&V, and system interaction testing

- Design and Production Reviews:

-ASC-E3 Long Lead Production Readiness Review (PRR) completed successfully November 2010 for heater head, displacer, and transition assemblies

- ASC-E3 Convertor-level PRR completed April 2011 followed by production approval June 2011

-ASC-F Final Design Review successfully competed February 2012

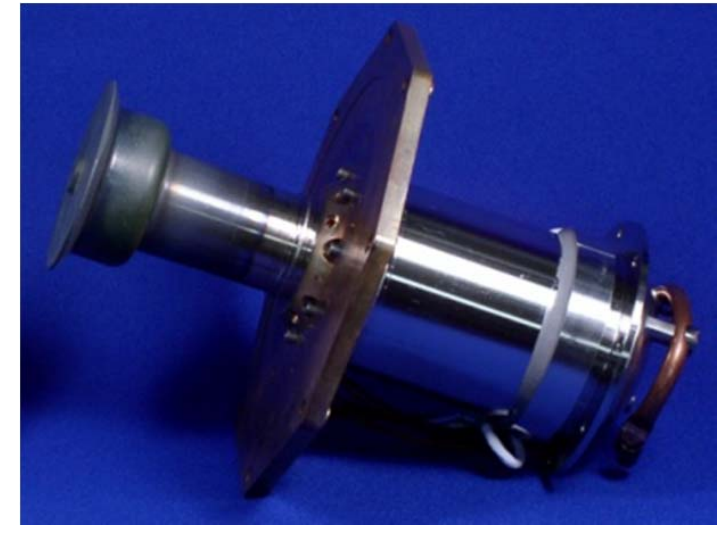

ASC-E3 and ASC-F based on the successful ASC-E2 


\section{Advancements in ASC-F and ASC-E3 Design (1 of 3)}

- Heater Head Assembly Non-Destructive Inspection

- Multiple quality checks to ensure acceptability and that hermeticity requirement is met

- Two new GRC techniques used for ASC-E3 \& -F heater heads

- Microfocus X-ray Computed Tomography (CT)

- Microfocus CT selected after assessing various other nondestructive inspection (NDI) techniques for ASC heater head geometry and requirements

- GRC system is one of the highest resolution systems in U.S.

- CT creates 3-D model of inspected object through computer processing and reconstruction of many 2-D digital x-ray images taken of object around axis of rotation

- Heater Head High Temperature Helium Leak Test

- High temperature leak test assesses hermeticity at pressure and temperature - originally used to demonstrate helium at temperature and pressure does not permeate heater head walls

- Uses a very tight crushed metallic seal between heater head open end and actively cooled mounting flange.

- System uses a residual gas analyzer and vacuum pressure gage for leak detection.

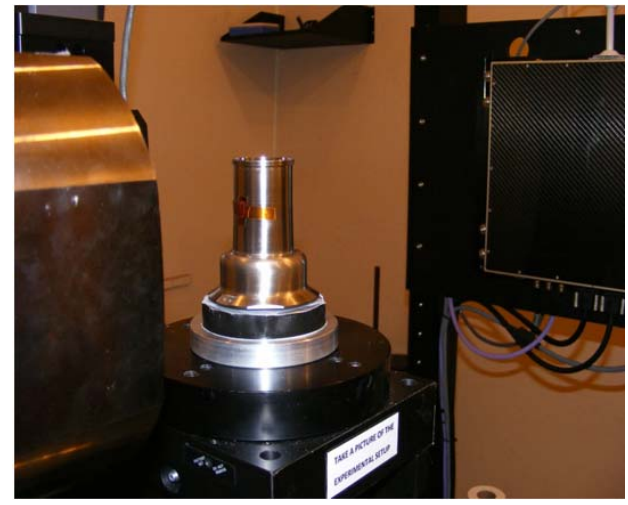

GRC Microfocus CT Setup

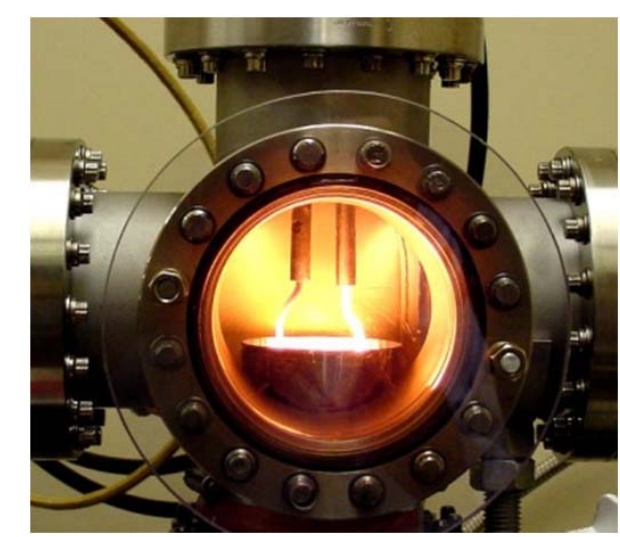

Permeation Rig now used for High Temp Leak Test 


\section{Advancements in ASC-F and ASC-E3 Design (2 of 3)}

- Cold Side Adapter Flange (CSAF) Attachment

- Weld used previously on ASC-E2 has been replaced by interference fit mechanical joint

- ASC-E3 pathfinding hardware revealed that the flight CSAF design resulted in poor weld joints that would have required additional development to resolve

- New interference fit joint, along with existing mechanical CSAF fasteners were demonstrated through analysis and qualification level vibration test of an ASC mock-up to meet structural requirements

- Thermal vacuum testing of new interference fit joint demonstrated acceptable thermal performance

- Alternator design update

- Based on ASC-E2 data, LM increased the ASC-F output voltage specification to improve controller design

- Only the ASC coil design was modified to avoid cascading design changes. The modification had negligible impact on ASC performance. 


\section{Advancements in ASC-F and ASC-E3 Design (3 of 3)}

- Regenerator production

- Production methods for high-temperature capable, oxidation resistant regenerator developed at GRC. GRC produced regenerators for earlier ASC-E and E2

- Sunpower has been developing in-house capability to produce regenerators and completed production verification. Sunpower producing all regenerators for ASC-E3 and $-\mathrm{F}$.

- Clean Room Processing

- ASC clean room production necessary to meet flight cleanliness and planetary protection requirements

- Requires significant updates to design and process documentation, and training of staff

- ASC-E3 Pair 2 is first hardware to be produced in Sunpower's new clean room facility

- Durability Testing

- ASC-E2 convertors were reworked to include some flight design changes and used to assess ASC durability including stop/start cycling, centrifugal acceleration, vibration induced contact, and overstroke assessment (Oriti IECEC presentation)
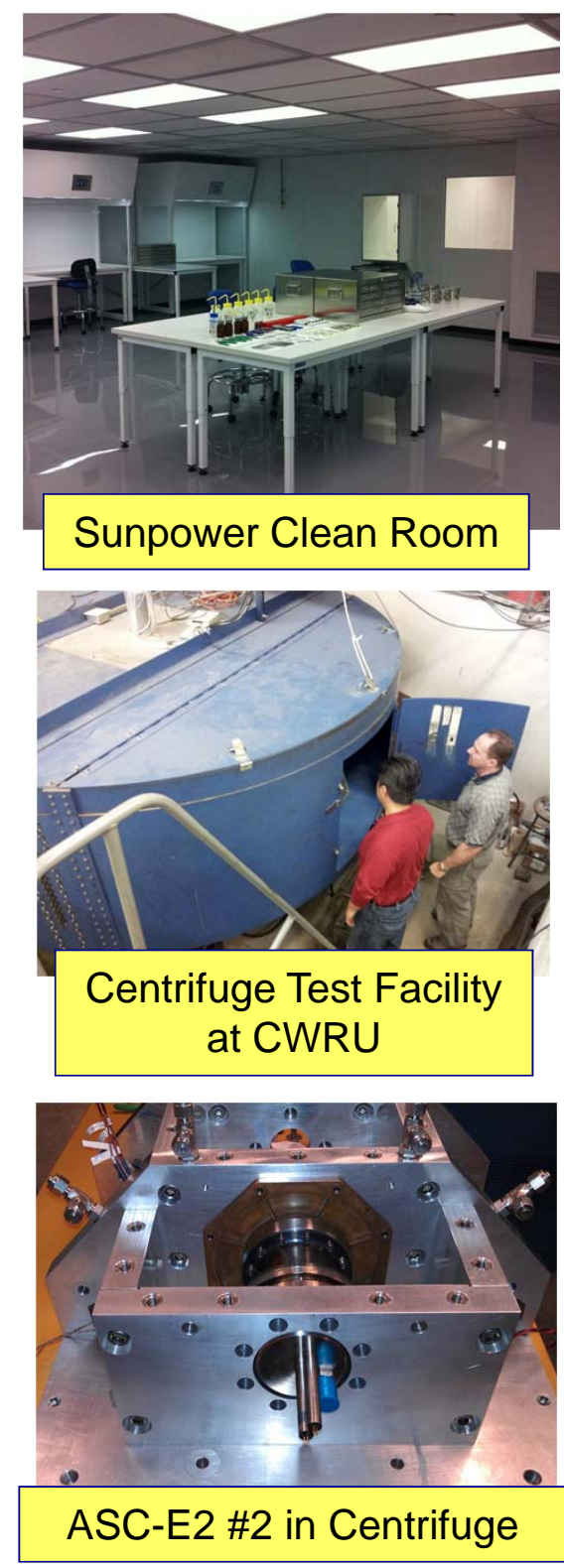


\section{ASC Status Highlights (1 of 2)}

- Sunpower relocated to new facility - Grand opening 8/2011

- Successfully demonstrated new CSAF interference fit

- Long Lead hardware for first two pairs of ASC-E3 completed including heater heads, displacers, \& transition assemblies

- Completed common ASC test setup design including structural support, insulation, heaters, cooling jacket, etc.

- Common setup used at different locations including Sunpower performance tests, and GRC independent validation tests

- Minimizes data variation and reduces tear-down and re-assembly time

- Continuous extended operation of convertors at GRC (as of June 2012)

- Total accumulated GRC ASC operating time is $>220,000$ hours

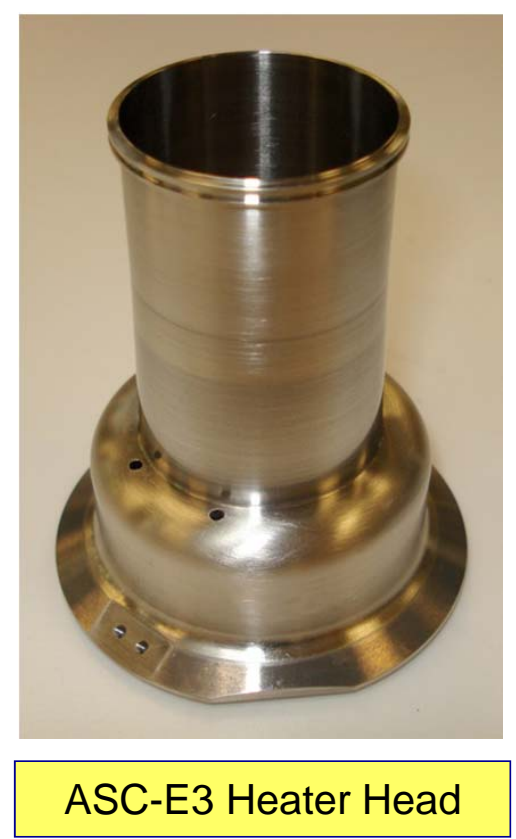

- Leading pair of ASC-0 units have $>25,000$ hours each

- The ASRG EU (with a pair of ASC-E) has $>27,000$ hours 


\section{ASC Status Highlights (2 of 2)}

- Sunpower ran initial ASC-E3 on 3/13/2012, demonstrating first operation of flight-like design. Initial non-hermetic configuration allows checkout of convertor with test piston, displacer and back pressure vessel

- ASC-E3 Pair 1 convertors completed variety of testing, have completed heater head weld and pressure vessel welds, and are being prepared for completion with hermetic sealing. Will then undergo performance tests and workmanship vibration tests

- ASC-E3 Pair 2 - Pathfinder for cleanroom processing have started assembly. Identifying process improvements in the clean room.

- Lessons learned on ASC-E3 Pair 1 and Pair 2 are being incorporated prior to flight build, reducing technical and schedule risk to ASC-F convertors

- Many components and subassemblies for remaining ASC-E3 and $-\mathrm{F}$ are complete and being prepared for assembly.
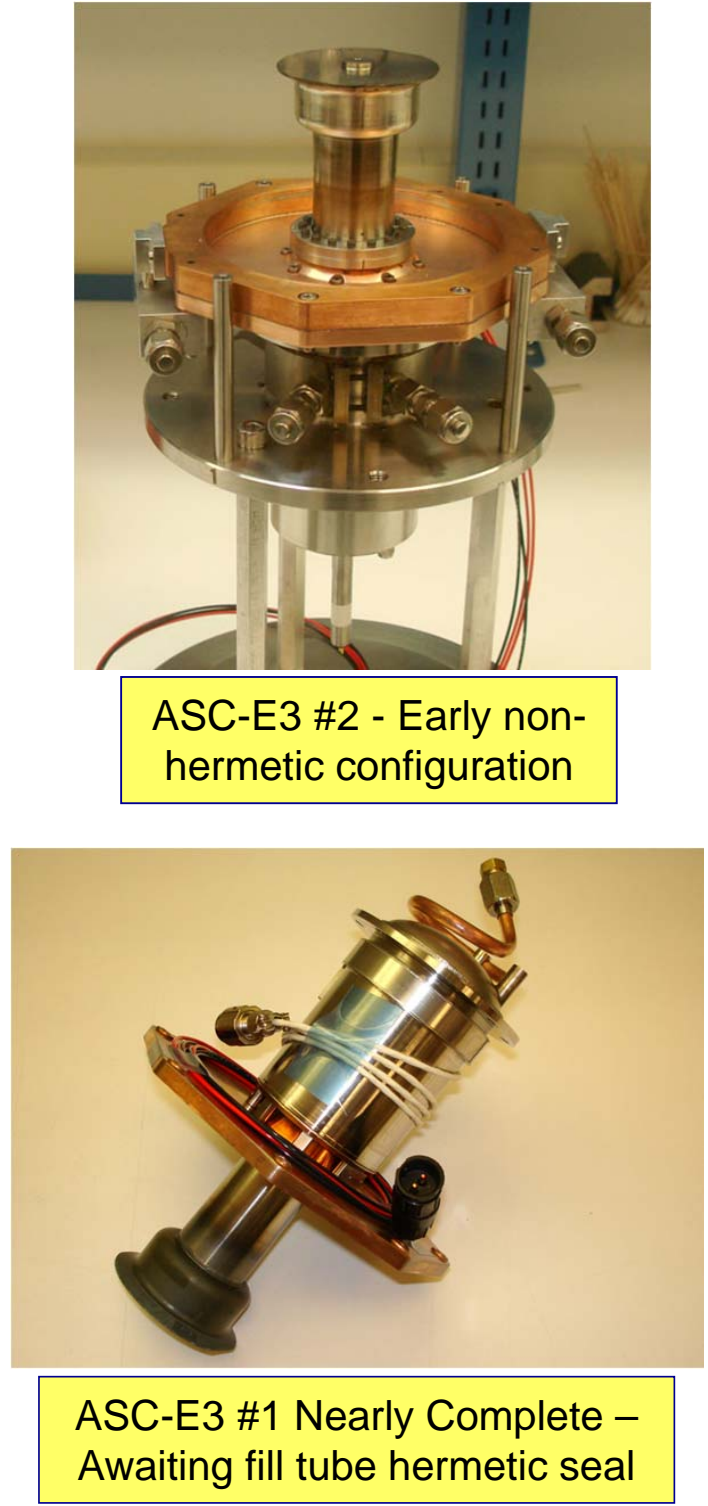


\section{Path Forward}

- ASC-E3 Pair 1 Delivery end of summer 2012 - After GRC acceptance testing, Pair 1 to be provided to Lockheed Martin for controller validation testing

- ASC-E3 Pair 2 will be completed in new Sunpower clean room facility with new cleanliness and planetary protection processes. Lessons learned being applied to flight convertors

- ASC-E3 second, third, and fourth pairs will all undergo a suite of planned tests at GRC including acceptance testing, extended operation, independent validation and verification tests, and system interaction tests

- The ASC-E3 are paving the way for flight production

- ASC-F/ASRG being prepared to support potential Discovery 12 mission 
